\title{
Incompatibility probability of random quantum measurements
}

\author{
Lin Zhang, ${ }^{1,2, *}$ Hua Xiang,,${ }^{3, \dagger}$ Xianqing Li-Jost,${ }^{2,}$ and Shao-Ming Fei ${ }^{2,4, \S}$ \\ ${ }^{1}$ Institute of Mathematics, Hangzhou Dianzi University, Hangzhou 310018, PR China \\ ${ }^{2}$ Max-Planck-Institute for Mathematics in the Sciences, Leipzig 04103, Germany \\ ${ }^{3}$ School of Mathematics and Statistics, Wuhan University, Wuhan 430072, PR China \\ ${ }^{4}$ School of Mathematical Sciences, Capital Normal University, Beijing 100048, China
}

\begin{abstract}
Incompatibility of quantum measurements is of fundamental importance in quantum mechanics. It is closely related to many nonclassical phenomena such as Bell nonlocality, quantum uncertainty relations, and quantum steering. We study the necessary and sufficient conditions of quantum compatibility for a given collection of $n$ measurements in $d$-dimensional space. From the compatibility criterion for two-qubit measurements, we compute the incompatibility probability of a pair of independent random measurements. For a pair of unbiased random qubit measurements, we derive that the incompatibility probability is exactly $\frac{3}{5}$. Detailed results are also presented in figures for pairs of general qubit measurements.
\end{abstract}

\section{INTRODUCTION}

Quantum theory has become the pillar of modern physics. Features such as non-locality [1], steering [2], entanglement [3, 4], contextuality [5], uncertainty [6] and coherence [7] distinguish quantum physics from classical physics. Among these features of quantum physics, the quantum incompatibility of quantum measurements forbids one from measuring two observables simultaneously exactly when they are incompatible. Quantum incompatibility can lead to many novel phenomena including measurement uncertainty relations [8], steerability [9] and nonlocality [10]. In the case of a pair of two-outcome measurements, the incompatibility is equivalent to Bell non-locality $[11,12]$, though measurement incompatibility does not imply Bell non-locality in general $[13,14]$.

Concerning quantum incompatibility, an important problem is the development of an effective method to judge whether a set of measurements is compatible (i.e., jointly measurable), which has received much attention [15-17]. The authors of [15] and [16] used the notion of free spectrahedra in the optimization theory to characterize the measurement compatibility (also known as the joint measurability). Due to the abstract construction of free spectahedra, characterization of incompatibility along this approach is not very operational. The authors of a recent study [17] presented a more operational way toward the characterization of quantum incompatibility for the case where both measurements have the same number of measurement outcomes.

The relations between quantum measurement incompatibility and quantum information processing have also been extensively investigated. In fact, it is shown that quantum incompatibility can be detected by a state discrimination task with partial intermediate information $[18,19]$. Looking at it from another perspective, every set of incompatible measurements provides an advantage over compatible ones in a suitably chosen quantum state discrimination task [20, 21].

\footnotetext{
* godyalin@163.com

† hxiang@whu.edu.cn

‡xli-jost@mis.mpg.de

$\S$ feishm@cnu.edu.cn
}

In [22] the separability probability problem has been addressed: What is the probability that a randomly given quantum state is entangled (or separable)? In order to answer this question, the authors proposed to calculate the volume of all separable bipartite states in a portion of the whole set of bipartite states [23] and [24]. The issue of probing entanglement and constructing a separable form in the context of separable states has also been addressed in [25, 26]. Nevertheless, even for the simplest case (i.e. two-qubit quantum states), computing the separability probability according to the HilbertSchmidt measure is still a challenging problem. Numerical simulations lead to intriguing formulas for separability probability [27]. It turned out that the geometric separability probability of two-qubit quantum systems is conjectured to be $\frac{8}{33}$, without proof up to now [28].

Motivated by the problem of separability probability, we ask what the probability is for a randomly given pair of measurements [positive operator valued measurements (POVMs)] to be incompatible. However, calculation of the incompatibility probability depends heavily on the criteria of incompatibility. For a pair of unbiased random qubit measurements with two measurement outcomes, we derive that the incompatibility probability is exactly $\frac{3}{5}$. The incompatibility probability for a pair of general qubit measurements is conjectured to be $\frac{1}{4}$ by numerical simulation. As for the case of a pair of twooutcome measurements, the incompatibility is equivalent to Bell non-locality [12], this fact suggests that $25 \%$ of pairs of qubit measurements can lead to Bell non-locality. If we are restricted to the use of pairs of unbiased qubit measurements, the fraction increases to $60 \%$.

In this paper, first we deal with the necessary and sufficient conditions of (in-)compatibility for a finite number of measurements with arbitrary finite outcomes. Then we investigate the geometry of the set of incompatible pairs of measurements. We compute the incompatibility probability: the ratio of the set of incompatible pairs of measurements versus the set of all pairs of measurements. 


\section{CHARACTERIZATION OF QUANTUM MEASUREMENT INCOMPATIBILITY}

For a positive integer $\ell$, denote $[\ell]:=\{1, \ldots, \ell\}$. We say that $\mathrm{M}=\left(M_{i_{1} \ldots i_{n}}\right)$ is an $n$-th Hermitian tensor if each $M_{i_{1} \ldots i_{n}}$ is a Hermitian operator acting on $d$-dimensional Hilbert space $\mathcal{H}_{d}$, where $i_{1} \in\left[k_{1}\right], \ldots, i_{n} \in\left[k_{n}\right]$. A POVM is represented by an $n$-th Hermitian tensor $\mathrm{M}=$ $\left(M_{i_{1} \ldots i_{n}}\right)$, where each $M_{i_{1} \ldots i_{n}}$ is positive semi-definite and $\sum M_{i_{1} \ldots i_{n}}=\mathbf{1}_{d}$, with $\mathbf{1}_{d}$ the identity operator on $\mathcal{H}_{d}$. The following $n$ POVMs $\mathrm{A}^{(1)}=\left(A_{i_{1}}^{(1)}\right), \ldots, \mathrm{A}^{(n)}=\left(A_{i_{n}}^{(n)}\right)$, with $i_{1} \in\left[k_{1}\right], \ldots, i_{n} \in\left[k_{n}\right]$, defined by

$$
A_{i_{1}}^{(1)} \stackrel{\text { def }}{=} \sum_{i_{2} \ldots i_{n}} M_{i_{1} \ldots i_{n}}, \ldots, A_{i_{n}}^{(n)} \stackrel{\text { def }}{=} \sum_{i_{1} \ldots i_{n-1}} M_{i_{1} \ldots i_{n}}
$$

are called the marginals of $\mathrm{M}$.

For $n$ given POVMs $\mathrm{A}^{(l)}(l \in[n])$ on $\mathcal{H}_{d}$, if there exists a POVM, $\mathrm{M}=\left(M_{i_{1} \ldots i_{n}}\right)$, where $i_{l} \in\left[k_{l}\right](l \in[n])$, such that $\mathrm{A}^{(l)}(l \in[n])$ are the marginals of $\mathrm{M}$, we say that the $n$ POVMs $\mathrm{A}^{(l)}(l \in[n])$ are compatible or jointly measurable, and $\mathrm{M}$ is called the joint measurement. Otherwise, they are called incompatible [16].

Generally, for given $n$ jointly measurable POVMs $\mathrm{A}^{(l)}$ $(l \in[n])$, their joint measurements are not unique. We denote $\mathcal{J}\left(\mathrm{A}^{(1)}, \ldots, \mathrm{A}^{(n)}\right)$ all the joint measurements for $n$ arbitrary given POVMs $\mathbf{A}^{(l)}(l \in[n])$. Then $\mathcal{J}\left(\mathbf{A}^{(1)}, \ldots, \mathbf{A}^{(n)}\right)=\emptyset$ if the $n$ POVMs $\mathbf{A}^{(l)}(l \in[n])$ are not jointly measurable. Thus $A^{(l)}(l \in[n])$ are jointly measurable if and only if $\mathcal{J}\left(\mathrm{A}^{(1)}, \ldots, \mathrm{A}^{(n)}\right) \neq \emptyset$.

Consider $n$ arbitrary probability vectors $\boldsymbol{p}^{(1)}=$ $\left(p_{i_{1}}^{(1)}\right), \ldots, \boldsymbol{p}^{(n)}=\left(p_{i_{n}}^{(n)}\right)$, where $i_{l} \in\left[k_{l}\right](l \in[n])$, such that all the components of $p^{(l)}$ are positive for all $l \in[n]$. Denote $\boldsymbol{p}^{(l)} \otimes \mathbf{1}_{d}$ a POVM with measurement operators given by $\left\{p_{i_{l}}^{(l)} \mathbf{1}_{d}\right\}$, where $i_{l} \in\left[k_{l}\right], l \in[n]$. Clearly, $\mathcal{J}\left(\boldsymbol{p}^{(1)} \otimes \mathbf{1}_{d}, \ldots, \boldsymbol{p}^{(n)} \otimes \mathbf{1}_{d}\right) \neq \emptyset$. Let $\mathbf{T}=\left(T_{i_{1} \ldots i_{n}}\right)$ be the $n$-th Hermitian tensor such that their $n$ marginals are given by $\boldsymbol{p}^{(1)} \otimes \mathbf{1}_{d}, \ldots, \boldsymbol{p}^{(n)} \otimes \mathbf{1}_{d}$,

$$
\sum_{i_{2}, \ldots, i_{k}} T_{i_{1} \ldots i_{n}}=p_{i_{1}}^{(1)} \mathbf{1}_{d}, \ldots, \sum_{i_{1}, \ldots, i_{n-1}} T_{i_{1} \ldots i_{n}}=p_{i_{n}}^{(n)} \mathbf{1}_{d} .
$$

Namely, $\boldsymbol{T} \in \mathcal{J}\left(\boldsymbol{p}^{(1)} \otimes \mathbf{1}_{d}, \ldots, \boldsymbol{p}^{(n)} \otimes \mathbf{1}_{d}\right)$. We have

Theorem 1. For $n$ POVMs $\mathrm{A}^{(l)}$ and $n$ probability vectors $\boldsymbol{p}^{(l)}$, set

$$
M_{i_{1} \ldots i_{n}}=\left(\prod_{\ell=1}^{n} p_{i_{\ell}}^{(\ell)}\right) \sum_{l=1}^{n} \frac{1}{p_{i_{l}}^{(l)}} A_{i_{l}}^{(l)}-(n-1) T_{i_{1} \ldots i_{n}} .
$$

Then $\mathrm{A}^{(l)}(l \in[n])$ are $n$ marginals of $\mathbf{M}=\left(M_{i_{1} \ldots i_{n}}\right)$, and $\mathbf{A}^{(l)}(l \in[n])$ are compatible if and only if for any collection of $n$ probability vectors $\boldsymbol{p}^{(l)}(l \in[n])$, there exists some $n$-th Hermitian tensor $T \in \mathcal{J}\left(\boldsymbol{p}^{(1)} \otimes \mathbf{1}_{d}, \ldots, \boldsymbol{p}^{(n)} \otimes \mathbf{1}_{d}\right)$ such that $\mathrm{M}$ is a POVM.
Proof. Define $\mathrm{G}=\left(G_{i_{1} \ldots i_{n}}\right)$ as follows:

$$
G_{i_{1} \ldots i_{n}} \stackrel{\text { def }}{=} \frac{\prod_{\ell=1}^{n} p_{i_{\ell}}^{(\ell)}}{n} \sum_{l=1}^{n} \frac{1}{p_{i_{l}}^{(l)}} A_{i_{l}}^{(l)} .
$$

It is directly verified that $\mathrm{G}$ is a POVM. Its $n$ marginals are given by

$$
\frac{1}{n} \mathrm{~A}^{(l)}+\left(1-\frac{1}{n}\right) \boldsymbol{p}^{(l)} \otimes \mathbf{1}_{d} \quad(l \in[n]) .
$$

Here we view each POVM as a column-block matrix and $\otimes$ stands for the Kronecker tensor product. That is,

$$
\mathbf{A}^{(l)}=\left(\begin{array}{c}
A_{1}^{(l)} \\
\vdots \\
A_{k_{l}}^{(l)}
\end{array}\right) \text { and } \boldsymbol{p}^{(l)} \otimes \mathbf{1}_{d}=\left(\begin{array}{c}
p_{1}^{(l)} \mathbf{1}_{d} \\
\vdots \\
p_{k_{l}}^{(l)} \mathbf{1}_{d}
\end{array}\right) .
$$

Apparently $G_{i_{1} \ldots i_{n}}$ is non-negative for all $i_{l} \in\left[k_{l}\right](l \in[n])$ by definition. Moreover, one has

$$
\sum_{i_{2}, \ldots, i_{n}} G_{i_{1} \ldots i_{n}}=\frac{1}{n} A_{i_{1}}^{(1)}+\frac{1}{n} p_{i_{1}}^{(1)} \mathbf{1}_{d}+\cdots+\frac{1}{n} p_{i_{1}}^{(1)} \mathbf{1}_{d},
$$

and hence $\sum_{i_{1}, \ldots, i_{n}} G_{i_{1} \ldots i_{n}}=\mathbf{1}_{d}$. This shows that $\frac{1}{n} \mathrm{~A}^{(1)}+$ $\left(1-\frac{1}{n}\right) \boldsymbol{p}^{(1)} \otimes \mathbf{1}_{d}$ is one of the marginals of $\mathrm{G}$. Other marginals can be obtained similarly. Furthermore, the $n$ th Hermitian tensor $n \mathrm{G}-(n-1) \mathbf{T}=\left(n G_{i_{1} \ldots i_{n}}-(n-\right.$ 1) $\left.T_{i_{1} \ldots i_{n}}\right)$ has $n$ marginals $\mathrm{A}^{(1)}, \ldots, \mathrm{A}^{(n)}$. Indeed, for instance, $\sum_{i_{2}, \ldots, i_{n}}\left(n G_{i_{1} \ldots i_{n}}-(n-1) T_{i_{1} \ldots i_{n}}\right)=A_{i_{1}}^{(1)}$. This completes the proof.

Theorem 1 also indicates that adding noise to the POVMs, i.e., taking convex combinations of the original measurement operators of these POVMs and the trivial measurement (the identity operator), can make the resulting new POVMs more compatible (jointly measurable).

In the following we consider the case of $d=n=2$. Let $\mathrm{A}=\left(A_{1}, A_{2}\right)$ and $\mathrm{B}=\left(B_{1}, B_{2}\right)$ be two POVMs on $\mathbb{C}^{2}$. By using the Bloch representation, we can generally write

$$
A_{i}=\frac{1}{2}\left[\left(1+(-1)^{i} a_{0}\right) \mathbf{1}_{2}+(-1)^{i} \boldsymbol{a} \cdot \boldsymbol{\sigma}\right], \quad i=1,2,
$$

where $\boldsymbol{\sigma}=\left(\sigma_{1}, \sigma_{2}, \sigma_{3}\right)$ with $\sigma_{i}, \mathrm{i}=1,2,3$, the Pauli matrices, and $\boldsymbol{a}$ is a three dimensional real vector satisfying $|\boldsymbol{a}| \leqslant 1-$ $\left|a_{0}\right|$ with $a_{0} \in[-1,1]$. Here $|\boldsymbol{a}|$ is referred to as the sharpness while $\left|a_{0}\right|$ the biasedness. Similarly,

$$
B_{j}=\frac{1}{2}\left[\left(1+(-1)^{j} b_{0}\right) \mathbf{1}_{2}+(-1)^{j} \boldsymbol{b} \cdot \boldsymbol{\sigma}\right], j=1,2,
$$

where $|\boldsymbol{b}| \leqslant 1-\left|b_{0}\right|$ with $b_{0} \in[-1,1]$. Choosing arbitrarily two probability vectors $\boldsymbol{p}=\left(p_{1}, p_{2}\right)$ and $\boldsymbol{q}=\left(q_{1}, q_{2}\right)$, we have from Eq. (1),

$$
\mathrm{M}(\mathrm{A}, \mathrm{B} ; \boldsymbol{p}, \boldsymbol{q} ; \mathrm{T})=\left(M_{i j}\right),
$$

where $M_{i j}=q_{j} A_{i}+p_{i} B_{j}-T_{i j}$, and $\mathbf{T}=\left(T_{i j}\right)$ satisfies that $T_{i 1}+T_{i 2}=p_{i} \mathbf{1}_{2}$ and $T_{1 j}+T_{2 j}=q_{j} \mathbf{1}_{2}$ for $i, j \in[2]$. 
We write $T$ in the block-matrix form:

$$
\mathbf{T}=\left(\begin{array}{cc}
X & p_{1} \mathbf{1}_{2}-X \\
q_{1} \mathbf{1}_{2}-X & X+\left(q_{2}-p_{1}\right) \mathbf{1}_{2}
\end{array}\right),
$$

where $p_{2}-q_{1}=q_{2}-p_{1}$, and $X$ is some $2 \times 2$ Hermitian matrix. Assume that $p_{1}=p$ and $q_{1}=q$, where $p, q \in[0,1]$. By Bloch representation, $X$ can be written as

$$
X=\frac{1}{2}\left[\left(1-x_{0}\right) \mathbf{1}_{2}-\boldsymbol{x} \cdot \boldsymbol{\sigma}\right], \quad\left(x_{0}, \boldsymbol{x}\right) \in \mathbb{R}^{4} .
$$

Note that $\mathrm{M}$ is a legal POVM if and only if $M_{i j} \geqslant 0$ for all $i, j$. In other words, $\mathrm{M}$ is a POVM if and only if

$$
\left\{\begin{array}{l}
|q \boldsymbol{a}+p \boldsymbol{b}-\boldsymbol{x}| \\
\quad \leqslant(q+p-1)-\left(q a_{0}+p b_{0}-x_{0}\right), \\
|\boldsymbol{x}+(1-q) \boldsymbol{a}-p \boldsymbol{b}| \\
\quad \leqslant(2-q-p)-\left(x_{0}+(1-q) a_{0}-p b_{0}\right), \\
|\boldsymbol{x}-q \boldsymbol{a}+(1-p) \boldsymbol{b}| \\
\quad \leqslant(2-q-p)-\left(x_{0}-q a_{0}+(1-p) b_{0}\right), \\
|(1-q) \boldsymbol{a}+(1-p) \boldsymbol{b}+\boldsymbol{x}| \\
\quad \leqslant(q+p-1)+\left(x_{0}+(1-q) a_{0}+(1-p) b_{0}\right) .
\end{array}\right.
$$

A question naturally arises is: What kind of relationship should be satisfied by the 8-tuple $\left(a_{0}, \boldsymbol{a}, b_{0}, \boldsymbol{b}\right)$ such that $\mathrm{A}$ and $\mathrm{B}$ are jointly measurable for any prescribed $\boldsymbol{p}$ and $\boldsymbol{q}$. Since T is $\boldsymbol{p}$ and $\boldsymbol{q}$ dependent, without loss of generality, we may take probability vectors $\boldsymbol{p}=\boldsymbol{q}=\left(\frac{1}{2}, \frac{1}{2}\right)$. Denote $\boldsymbol{u}=\boldsymbol{a}+\boldsymbol{b}$, $\boldsymbol{v}=\boldsymbol{a}-\boldsymbol{b} ; \alpha=a_{0}+b_{0}, \beta=a_{0}-b_{0}$, and $\boldsymbol{y}=2 \boldsymbol{x}, y_{0}=2 x_{0}$. Now for a pair of unbiased observables $\mathrm{A}$ and B, i.e., $a_{0}=$ $b_{0}=0$, one has $\alpha=\beta=0$ and the solution set of the above four inequalities is not empty if and only if $y_{0} \geqslant|\boldsymbol{u}|$ and $2-$ $y_{0} \geqslant|\boldsymbol{v}|$, i.e., $y_{0}$ in the closed interval $[|\boldsymbol{u}|, 2-|\boldsymbol{v}|]$. This amounts to saying that $|\boldsymbol{u}| \leqslant 2-|\boldsymbol{v}|$. Therefore A and B are jointly measurable if and only if $|\boldsymbol{a}+\boldsymbol{b}|+|\boldsymbol{a}-\boldsymbol{b}| \leqslant 2$, which is just the result obtained in [29]. For a general pair of qubit observables, the following result [30] answers this question. For a pair of qubit observables $A$ and $B$ in Eq. (2) and Eq. (3), respectively, $A$ and $B$ are compatible if and only if the following inequality holds:

$$
\begin{aligned}
& \left(1-h\left(a_{0}, \boldsymbol{a}\right)^{2}-h\left(b_{0}, \boldsymbol{b}\right)^{2}\right)\left(1-\frac{a_{0}^{2}}{h\left(a_{0}, \boldsymbol{a}\right)^{2}}-\frac{b_{0}^{2}}{h\left(b_{0}, \boldsymbol{b}\right)^{2}}\right) \\
& \leqslant\left(\langle\boldsymbol{a}, \boldsymbol{b}\rangle-a_{0} b_{0}\right)^{2},
\end{aligned}
$$

where $h\left(x_{0}, \boldsymbol{x}\right)=\frac{\sqrt{\left(1+x_{0}\right)-|\boldsymbol{x}|^{2}}+\sqrt{\left(1-x_{0}\right)-|\boldsymbol{x}|^{2}}}{2}$ for $x_{0} \in$ $[-1,1]$ and $|\boldsymbol{x}| \leqslant 1-\left|x_{0}\right|$.

Based on this result, in what follows, we analyze the incompatibility probability of random qubit measurements.

\section{INCOMPATIBILITY PROBABILITY OF RANDOM QUBIT MEASUREMENTS}

We now consider the following question. Let $\Theta_{d, n}$ be the set of all pairs $(\mathrm{A}, \mathrm{B})$ of POVMs with $n$ measurement operators each, with $\mathrm{A}=\left(A_{i}\right)_{i=1}^{n}$ and $\mathrm{B}=\left(B_{j}\right)_{j=1}^{n}$ acting on $\mathbb{C}^{d}$. Denote $\Theta_{d, n}^{\mathrm{NJM}}$ and $\Theta_{d, n}^{\mathrm{JM}}$ the set of all incompatible and compatible pairs of POVMs from $\Theta_{d, n}$, respectively. Namely, $\Theta_{d, n}^{\mathrm{JM}}=\Theta_{d, n} \backslash \Theta_{d, n}^{\mathrm{NJM}}$. Let $\operatorname{vol}\left(\Theta_{d, n}^{\mathrm{NJM}}\right)$ and
$\operatorname{vol}\left(\Theta_{d, n}\right)$ be the volumes of $\Theta_{d, n}^{\mathrm{NJM}}$ and $\Theta_{d, n}$, respectively. We would like to know the geometric probability of incompatibility, $\operatorname{Pr}\left[\Theta_{d, n}^{\mathrm{NJM}}\right]=\operatorname{vol}\left(\Theta_{d, n}^{\mathrm{NJM}}\right) / \operatorname{vol}\left(\Theta_{d, n}\right)$. This question heavily depends on the criteria of compatibility. We treat this problem below for the case of qubit POVMs.

In the following we study the geometric probability of incompatibility for fixed $\left(a_{0}, b_{0}\right)$. For fixed $\left(a_{0}, b_{0}\right) \in[-1,1] \times[-1,1]$ in POVMs Eq. (2) and Eq. (3), we denote $\Theta_{2,2}\left(a_{0}, b_{0}\right)$ the section of $\Theta_{2,2}$ at $\left(a_{0}, b_{0}\right)$, and similarly for $\Theta_{2,2}^{\mathrm{NJM}}\left(a_{0}, b_{0}\right)$ and $\Theta_{2,2}^{\mathrm{JM}}\left(a_{0}, b_{0}\right)$. We consider the parameterized probabilities: $\operatorname{Pr}\left[\Theta_{2,2}^{\mathrm{NJM}}\left(a_{0}, b_{0}\right)\right]=\operatorname{vol}\left(\Theta_{2,2}^{\mathrm{NJM}}\left(a_{0}, b_{0}\right)\right) / \operatorname{vol}\left(\Theta_{2,2}\left(a_{0}, b_{0}\right)\right)$ and $\operatorname{Pr}\left[\Theta_{2,2}^{\mathrm{JM}}\left(a_{0}, b_{0}\right)\right]=1-\operatorname{Pr}\left[\Theta_{2,2}^{\mathrm{NJM}}\left(a_{0}, b_{0}\right)\right]$. Note that the parameters $\boldsymbol{a}$ and $\boldsymbol{b}$ in $\Theta_{2,2}\left(a_{0}, b_{0}\right)$ satisfy the constraints $|\boldsymbol{a}| \leqslant 1-\left|a_{0}\right|$ and $|\boldsymbol{b}| \leqslant 1-\left|b_{0}\right|$. We have

$$
\operatorname{vol}\left(\Theta_{2,2}\left(a_{0}, b_{0}\right)\right)=\left(\frac{4 \pi}{3}\right)^{2}\left(1-\left|a_{0}\right|\right)^{3}\left(1-\left|b_{0}\right|\right)^{3} .
$$

It suffices to calculate the volume $\operatorname{vol}\left(\Theta_{2,2}^{\mathrm{NJM}}\left(a_{0}, b_{0}\right)\right)$.

\section{A. The case for unbiased measurements: $\left(a_{0}, b_{0}\right)=(0,0)$}

We first consider the unbiased POVMs $A$ and B, i.e., $\left(a_{0}, b_{0}\right)=(0,0)$, determined by the vectors $\boldsymbol{a}$ and $\boldsymbol{b}$, respectively. In this case Eq. (4) gives rise to the condition that $A$ and B are incompatible: $f(\boldsymbol{a}, \boldsymbol{b}):=|\boldsymbol{a}|^{2}+|\boldsymbol{b}|^{2}-s^{2}>1$, where $s=\langle\boldsymbol{u}, \boldsymbol{v}\rangle \in[-1,1]$. This condition $f(\boldsymbol{a}, \boldsymbol{b})>1$ is equivalent to $g(\boldsymbol{a}, \boldsymbol{b}):=|\boldsymbol{a}+\boldsymbol{b}|+|\boldsymbol{a}-\boldsymbol{b}|>2$ (see Appendix A).

In stead of calculating the volume $\operatorname{vol}\left(\Theta_{2,2}^{\mathrm{NJM}}(0,0)\right)$, here we can also consider $\boldsymbol{a}$ and $\boldsymbol{b}$ as random vectors with probability distribution $\mathrm{d} \omega=p(\boldsymbol{a}) p(\boldsymbol{b})[\mathrm{d} \boldsymbol{a}][\mathrm{d} \boldsymbol{b}]$ given by [31],

$$
\mathrm{d} \omega=\left(\frac{3}{4 \pi}\right)^{2} a^{2} b^{2} \delta(1-|\boldsymbol{u}|) \delta(1-|\boldsymbol{v}|) \mathrm{d} a \mathrm{~d} b[\mathrm{~d} \boldsymbol{u}][\mathrm{d} \boldsymbol{v}],(5)
$$

where $\boldsymbol{a}=a \boldsymbol{u}$ and $\boldsymbol{b}=b \boldsymbol{v}$ with $a=|\boldsymbol{a}| \in[0,1], b=|\boldsymbol{b}| \in$ $[0,1]$, and $|\boldsymbol{u}|=|\boldsymbol{v}|=1$.

Denote the whole domain corresponding to $\Theta_{2,2}(0,0)$ by $\widetilde{\Omega}=\left\{(\boldsymbol{a}, \boldsymbol{b}) \in \mathbb{R}^{3} \times \mathbb{R}^{3}:|\boldsymbol{a}| \leqslant 1\right.$ and $\left.|\boldsymbol{b}| \leqslant 1\right\}$ and the domain corresponding to $\Theta_{2,2}^{\mathrm{NJM}}(0,0)$ by the following

$$
\begin{aligned}
\widetilde{\Omega}_{\mathrm{NJM}} & =\left\{(\boldsymbol{a}, \boldsymbol{b}) \in \mathbb{R}^{6}: f(\boldsymbol{a}, \boldsymbol{b})>1 \wedge|\boldsymbol{a}| \leqslant 1 \wedge|\boldsymbol{b}| \leqslant 1\right\} \\
& =\left\{(\boldsymbol{a}, \boldsymbol{b}) \in \mathbb{R}^{6}: g(\boldsymbol{a}, \boldsymbol{b})>2 \wedge|\boldsymbol{a}| \leqslant 1 \wedge|\boldsymbol{b}| \leqslant 1\right\} .
\end{aligned}
$$

It is easily verified that $\int_{\widetilde{\Omega}} \mathrm{d} \omega=1$. The problem is to calculate $\int_{\widetilde{\Omega}_{\mathrm{NJM}}} \mathrm{d} \omega$.

The condition $f(\boldsymbol{a}, \boldsymbol{b})>1$ or $g(\boldsymbol{a}, \boldsymbol{b})>2$ can be expressed as $s^{2}<a^{-2}+b^{-2}-(a b)^{-2}$, which can be rewritten as

$$
\left\{\begin{array}{l}
a \in(0,1), b \in\left(\sqrt{1-a^{2}}, 1\right), \\
s \in\left(-\frac{\sqrt{a^{2}+b^{2}-1}}{a b}, \frac{\sqrt{a^{2}+b^{2}-1}}{a b}\right) .
\end{array}\right.
$$

The joint probability density function of such a 3-tuple $(a, b, s) \in[0,1]^{2} \times[-1,1]$ is given by

$$
p(a, b, s)=3 a^{2} \times 3 b^{2} \times \frac{1}{2}=\frac{9}{2} a^{2} b^{2} .
$$


Denote $\Omega_{\mathrm{NJM}}=\left\{(a, b, s) \in \Omega: s^{2}<a^{-2}+b^{-2}-(a b)^{-2}\right\}$, i.e., all 3-tuples $(a, b, s)$ corresponding to $f(\boldsymbol{a}, \boldsymbol{b})>1$ [or $g(\boldsymbol{a}, \boldsymbol{b})>2]$. Now the 3-tuple $(a, b, s) \in \Omega_{\mathrm{NJM}}$ if and only if the conditions in Eq. (6) are satisfied. In fact, $(a, b, s) \in \Omega_{\mathrm{NJM}}$ can also be rewritten as

$$
s \in(-1,1), \quad a \in(0,1), \quad b \in\left(\sqrt{\frac{1-a^{2}}{1-a^{2} s^{2}}}, 1\right) .
$$

Now the problem of calculating $\int_{\widetilde{\Omega}_{\mathrm{NJM}}} \mathrm{d} \omega$ is reduced to the calculation of $\int_{\Omega_{\mathrm{NJM}}} p(a, b, s) \mathrm{d} a \mathrm{~d} b \mathrm{~d} s$. We have

$$
\int_{\Omega_{\mathrm{NJM}}} p(a, b, s) \mathrm{d} a \mathrm{~d} b \mathrm{~d} s=\frac{3}{5} .
$$

Therefore, we have

Theorem 2. For a pair of random unbiased qubit POVMs $A$ and $B$, generated by Eq. (5) via Bloch representation, the incompatibility probability is given by

$$
\operatorname{Pr}\left[\Theta_{2,2}^{\mathrm{NJM}}(0,0)\right]=\frac{3}{5} .
$$

Remark. Equation (7) can also be derived by calculating the volume $\operatorname{vol}\left(\Theta_{2,2}^{\mathrm{NJM}}(0,0)\right)$. Using the Lebesgue measure, we have $[\mathrm{d} \boldsymbol{a}]=a^{2} \mathrm{~d} a \times \delta(1-|\boldsymbol{u}|)[\mathrm{d} \boldsymbol{u}]$. The Lebesgue volume of $\widetilde{\Omega}$ is given by $\operatorname{vol}(\widetilde{\Omega})=\frac{(4 \pi)^{2}}{9}$. Thus $\widetilde{\Omega}_{\mathrm{NJM}}$ can be expressed as

$$
\left\{(a \boldsymbol{u}, b \boldsymbol{v}) \in \widetilde{\Omega}: a \in(0,1), b \in\left(\sqrt{1-a^{2}}, 1\right), s \in\left(-\frac{\sqrt{a^{2}+b^{2}-1}}{a b}, \frac{\sqrt{a^{2}+b^{2}-1}}{a b}\right)\right\} .
$$

Then

$$
\begin{aligned}
\operatorname{vol}\left(\widetilde{\Omega}_{\mathrm{NJM}}\right) & =\int_{\widetilde{\Omega}_{\mathrm{NJM}}}[\mathrm{d} \boldsymbol{a}][\mathrm{d} \boldsymbol{b}]=N_{3}^{2} \int_{0}^{1} \mathrm{~d} a a^{2} \int_{\sqrt{1-a^{2}}}^{1} \mathrm{~d} b b^{2} \int_{-\frac{\sqrt{a^{2}+b^{2}-1}}{a b}}^{\frac{\sqrt{a^{2}+b^{2}-1}}{a b}} p_{3}(s) \mathrm{d} s \\
& =\frac{(4 \pi)^{2}}{2} \int_{0}^{1} \mathrm{~d} a a^{2} \int_{\sqrt{1-a^{2}}}^{1} \mathrm{~d} b b^{2} \int_{-\frac{\sqrt{a^{2}+b^{2}-1}}{a b}}^{\frac{\sqrt{a^{2}+b^{2}-1}}{a b}} \mathrm{~d} s \\
& =(4 \pi)^{2} \int_{0}^{1} \mathrm{~d} a a^{2} \int_{\sqrt{1-a^{2}}}^{1} \mathrm{~d} b b^{2} \frac{\sqrt{a^{2}+b^{2}-1}}{a b} \\
& =(4 \pi)^{2} \int_{0}^{1} \mathrm{~d} a a^{2} \frac{a^{2}}{3} \\
& =\frac{(4 \pi)^{2}}{15}
\end{aligned}
$$

where $N_{3}=4 \pi$ and $p_{3}(s)=\frac{1}{2}$. Note that $p_{3}(s)$ is just the case of $p_{m}(s)$ for $m=3$, and $p_{m}(s)$ is given by (see Appendix B)

$$
p_{m}(s)=\frac{1}{N_{m}^{2}} \int_{\mathbb{R}^{m} \times \mathbb{R}^{m}} \delta(s-\langle\boldsymbol{u}, \boldsymbol{v}\rangle) \delta(1-|\boldsymbol{u}|) \delta(1-|\boldsymbol{v}|)[\mathrm{d} \boldsymbol{u}][\mathrm{d} \boldsymbol{v}] .
$$

Therefore, the geometric probability of incompatibility is given by $\operatorname{vol}\left(\widetilde{\Omega}_{\mathrm{NJM}}\right) / \operatorname{vol}(\widetilde{\Omega})=3 / 5$.

As $\mathrm{A}$ and $\mathrm{B}$ are incompatible if $f(\boldsymbol{a}, \boldsymbol{b})>1$ [or $g(\boldsymbol{a}, \boldsymbol{b})>$ $2]$, it is also interesting to calculate analytically the expectations $\mathbb{E}[f(\boldsymbol{a}, \boldsymbol{b})]$ and $\mathbb{E}[g(\boldsymbol{a}, \boldsymbol{b})]$ of $f(\boldsymbol{a}, \boldsymbol{b})$ and $g(\boldsymbol{a}, \boldsymbol{b})$, respec- tively. By direct computation we have

$$
\begin{aligned}
\mathbb{E}[f(\boldsymbol{a}, \boldsymbol{b})] & =\int_{\Omega_{\mathrm{NJM}}} f(a, b, s) p(a, b, s) \mathrm{d} a \mathrm{~d} b \mathrm{~d} s \\
& =\frac{27}{25}>1
\end{aligned}
$$

and, similarly, $\mathbb{E}[g(\boldsymbol{a}, \boldsymbol{b})]=\frac{72}{35}>2$. These results are consistent with Eq. (7): two randomly selected measurements $A$ and $\mathrm{B}$ are most probably incompatible.

$$
\text { B. The case }\left(a_{0}, b_{0}\right)=(\lambda, 0) \text { for } \lambda \in(-1,1)
$$

The case $\left(a_{0}, b_{0}\right)=(\lambda, 0)$, where $\lambda \in(-1,1)$, corresponds to the case where $A$ is a biased measurement and $B$ is an unbiased one. In this case $\Theta_{2,2}^{\mathrm{NJM}}(\lambda, 0)$ can be parameterized as the set $\widetilde{\Theta}_{2,2}^{\mathrm{NJM}}(\lambda, 0)$ such that 


$$
\begin{aligned}
& b \in(\sqrt{|\lambda|}, 1), \quad s \in\left(-\sqrt{\frac{b^{2}-|\lambda|}{b^{2}(1-|\lambda|)}}, \sqrt{\frac{b^{2}-|\lambda|}{b^{2}(1-|\lambda|)}}\right), \\
& a \in\left(\sqrt{\frac{b^{2}-b^{4}-b^{2} s^{2}+b^{4} s^{2}-|\lambda|^{2}+b^{2}|\lambda|^{2}+b^{2} s^{2}|\lambda|^{2}-b^{4} s^{2}|\lambda|^{2}}{b^{2}\left(1-s^{2}\right)\left(1-b^{2} s^{2}\right)}}, 1-|\lambda|\right) .
\end{aligned}
$$

Thus,

$$
\operatorname{Pr}\left[\Theta_{2,2}^{\mathrm{NJM}}(\lambda, 0)\right]=\frac{9}{2}(1-|\lambda|)^{-3} \int_{\widetilde{\Theta}_{2,2}^{\mathrm{NJM}}(\lambda, 0)} a^{2} b^{2} \mathrm{~d} b \mathrm{~d} s \mathrm{~d} a .
$$

By numerical computation it can be shown that $\operatorname{Pr}\left[\Theta_{2,2}^{\mathrm{NJM}}(\lambda, 0)\right]$ decreases when $|\lambda| \in[0,1)$ increases. Namely for larger $|\lambda|$, randomly selected A and B are most probably compatible.

\section{The case of general $\left(a_{0}, b_{0}\right)$}

Generally, $\Theta_{2,2}$ can be identified as $\left(a_{0}, a \boldsymbol{u}, b_{0}, b \boldsymbol{v}\right)$ such that $\left|a_{0}\right|+a \leqslant 1$ with $a_{0} \in[-1,1], a \in[0,1]$ and $\left|b_{0}\right|+b \leqslant 1$ with $b_{0} \in[-1,1], b \in[0,1] . \Theta_{2,2}^{\mathrm{NJM}}$ is a subset of $\Theta_{2,2}$ and can be identified as the set that Eq. (4) is violated.

Denote $V=\left\{\left(x_{0}, \boldsymbol{x}\right) \in \mathbb{R}^{4}:\left|x_{0}\right|+|\boldsymbol{x}| \leqslant 1\right.$ for $\left.x_{0} \in[-1,1]\right\}$. We have

$$
\begin{aligned}
\operatorname{vol}(V) & =\int_{-1}^{1} \mathrm{~d} x_{0} \int_{\left\{\boldsymbol{x} \in \mathbb{R}^{3}:|\boldsymbol{x}| \leqslant 1-\left|x_{0}\right|\right\}}[\mathrm{d} \boldsymbol{x}] \\
& =\int_{-1}^{1} \frac{4 \pi\left(1-\left|x_{0}\right|\right)^{3}}{3} \mathrm{~d} x_{0}=\frac{2 \pi}{3} .
\end{aligned}
$$

It is easily seen that $\operatorname{vol}\left(\Theta_{2,2}\right)=\operatorname{vol}(V)^{2}=\left(\frac{2 \pi}{3}\right)^{2}$. Thus the incompatibility probability is given by

$$
\operatorname{Pr}\left[\Theta_{2,2}^{\mathrm{NJM}}\right]=\frac{\operatorname{vol}\left(\Theta_{2,2}^{\mathrm{NJM}}\right)}{\operatorname{vol}\left(\Theta_{2,2}\right)} .
$$

The volume of $\Theta_{2,2}^{\mathrm{NJM}}$ can be obtained as follows by numerical calculation (see Appendix $\mathrm{C}$ ): $\operatorname{vol}\left(\Theta_{2,2}^{\mathrm{NJM}}\right) \doteq 1.09662$, which is approximately $\frac{\pi^{2}}{9}$. We conjecture that the incompatibility probability of a pair of random qubit measurements $A$ and $B$, generated by $\left(a_{0}, \boldsymbol{a}\right)$ and $\left(b_{0}, \boldsymbol{b}\right)$ in Eq. (4), is given by

$$
\operatorname{Pr}\left[\Theta_{2,2}^{\mathrm{NJM}}\right]=\frac{1}{4} .
$$

Moreover, the following result can be found: if $\left|a_{0}\right|=$ $\left|b_{0}\right| \geqslant \frac{1}{2}$, then $\Theta_{2,2}^{\mathrm{NJM}}\left(a_{0}, b_{0}\right)=\emptyset$, i.e., $\mathrm{A}$ and $\mathrm{B}$ are compatible, $\operatorname{Pr}\left[\Theta_{2,2}^{\mathrm{NJM}}\left(a_{0}, b_{0}\right)\right]=0$.

More detailed computational results on the incompatibility probability $\operatorname{Pr}\left[\Theta_{2,2}^{\mathrm{NJM}}\left(a_{0}, b_{0}\right)\right]$ are displayed in Figs. 1-3. From the Fig. 1 we observe that outside the curve $\left|a_{0}\right|+\left|b_{0}\right|=$ 1 , the incompatibility probability is 0 . It increases smoothly towards the origin where it attains the peak value $\frac{3}{5}$. Figure 2 shows the contours of Fig. 1, displaying isolines of the incompatibility probability. Figure 3 is one quarter of Fig. 1, corresponding to the parameter regions $a_{0} \geqslant 0$ and $b_{0} \geqslant 0$.

FIG. 1. The incompatibility probability $\operatorname{Pr}\left[\Theta_{2,2}^{\mathrm{NJM}}\left(a_{0}, b_{0}\right)\right]$ of a pair of qubit measurements $\mathrm{A}$ and $\mathrm{B},\left(a_{0}, b_{0}\right) \in[-1,1]^{2}$.

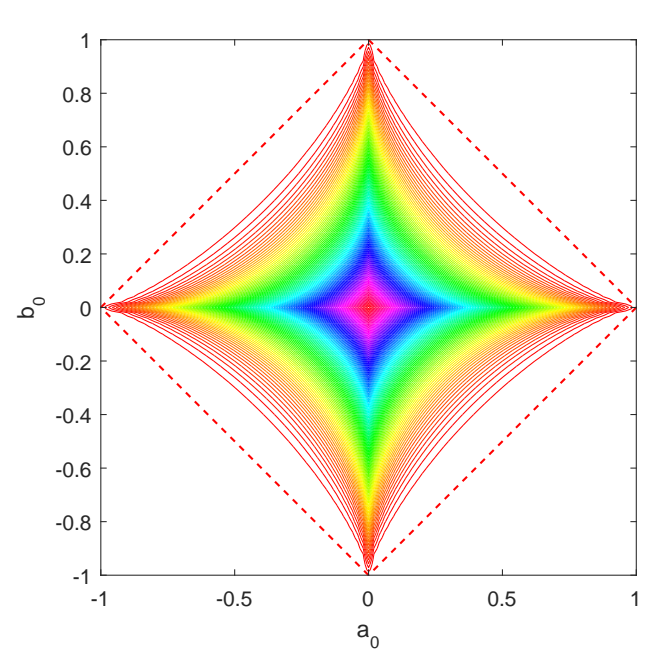

FIG. 2. The contours of the incompatibility probability $\operatorname{Pr}\left[\Theta_{2,2}^{\mathrm{NJM}}\left(a_{0}, b_{0}\right)\right]$ corresponding to Fig. 1.

\section{CONCLUSIONS}

We have dealt with the necessary and sufficient conditions of incompatibility for a finite number of measurements with arbitrary finite outcomes. Our approach toward quantum incompatibility covers essentially the theoretic framework of [17] and connects with the results in [29] and [30] in the 


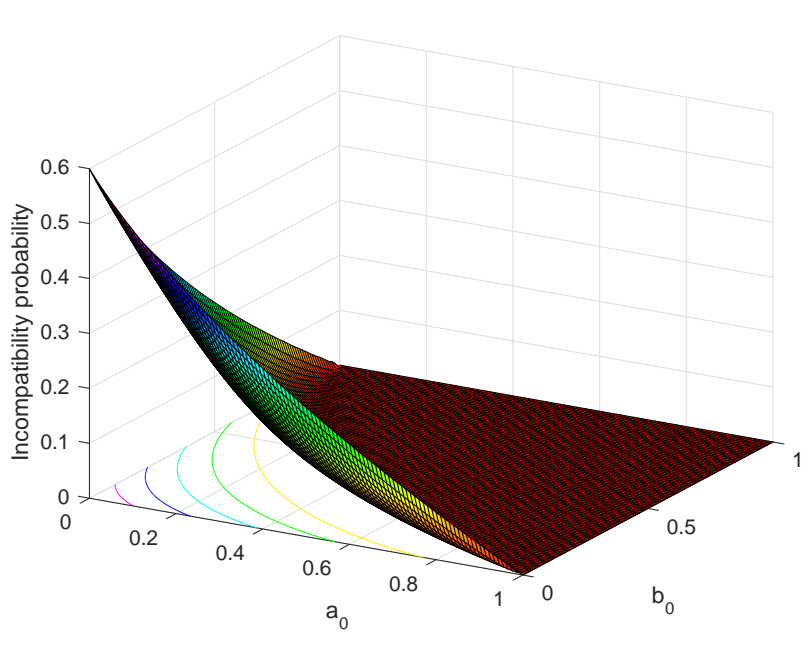

FIG. 3. The incompatibility probability $\operatorname{Pr}\left[\Theta_{2,2}^{\mathrm{NJM}}\left(a_{0}, b_{0}\right)\right]$ of a pair of qubit measurements $\mathrm{A}$ and $\mathrm{B}$ for $a_{0} \geqslant 0$ and $b_{0} \geqslant 0$.

case of qubit measurements with two-outcomes. Based on the necessary and sufficient conditions of compatibility for qubit measurements, we have analytically worked out the incompatibility probability of a pair of unbiased qubit measurements. The incompatibility probability of one unbiased and one biased qubit measurements, together with a pair of general qubit measurements, has also been investigated by analytical derivations and numerical calculations. These results may highlight studies on topics such as Bell nonlocality, quantum uncertainty and quantum steering. In fact, our results suggest that, the possibility of a pair of unbiased qubit measurements leading to Bell nonlocality is $60 \%$, which is larger than the $25 \%$ for general qubit measurements. Our results also indicate that the class of qubit unbiased measurements is the best choice for studying the connection between quantum measurement incompatibility and Bell non-locality $[13,14]$. It would also be interesting to design a schematic experiment to test the results. For such experimental verification, one needs to construct random gates to implement a random pair of POVMs $A$ and $B$ under a specified distribution. Besides qubit measurements, it is also interesting to estimate the incompatibility probability of a pair of high-dimensional measurements by using the convex geometry and probabilistic tools $[32,33]$.

\section{ACKNOWLEDGMENTS}

LZ would like to thank Xiao-Ming Lu for his comments on the revision of present manuscript. This research is supported by National Natural Science Foundation of China under Grant Nos. 11971140, 11701259, 11675113, Beijing Municipal Commission of Education (KZ201810028042), and Beijing Natural Science Foundation (Z190005). LZ is also supported by Zhejiang Provincial Natural Science Foundation of China under Grant no. LY17A010027. Appendix A: POOF OF THE EQUIVALENCE OF $f(\boldsymbol{a}, \boldsymbol{b})>1$
AND $g(\boldsymbol{a}, \boldsymbol{b})>2$

This result is mentioned in [30] without proof. We provide below the detailed proof for completeness. We first present the following proposition.

Proposition A.1. If $\boldsymbol{a}, \boldsymbol{b} \in \mathbb{R}^{3}$ with $|\boldsymbol{a}| \leqslant 1$ and $|\boldsymbol{b}| \leqslant 1$, then $|\boldsymbol{a}|^{2}+|\boldsymbol{b}|^{2} \leqslant 1+\langle\boldsymbol{a}, \boldsymbol{b}\rangle^{2}$ if and only if $|\boldsymbol{a}+\boldsymbol{b}|+|\boldsymbol{a}-\boldsymbol{b}| \leqslant 2$.

Proof. If $|\boldsymbol{a}|^{2}+|\boldsymbol{b}|^{2} \leqslant 1+\langle\boldsymbol{a}, \boldsymbol{b}\rangle^{2}$ for $|\boldsymbol{a}| \leqslant 1$ and $|\boldsymbol{b}| \leqslant 1$, then $|\boldsymbol{a}|^{2}+|\boldsymbol{b}|^{2} \pm 2\langle\boldsymbol{a}, \boldsymbol{b}\rangle \leqslant 1 \pm 2\langle\boldsymbol{a}, \boldsymbol{b}\rangle+\langle\boldsymbol{a}, \boldsymbol{b}\rangle^{2}$. That is, $|\boldsymbol{a} \pm \boldsymbol{b}| \leqslant 1 \pm\langle\boldsymbol{a}, \boldsymbol{b}\rangle$. Then $|\boldsymbol{a}+\boldsymbol{b}|+|\boldsymbol{a}-\boldsymbol{b}| \leqslant 2$.

Now conversely, if $|\boldsymbol{a}+\boldsymbol{b}|+|\boldsymbol{a}-\boldsymbol{b}| \leqslant 2$ for $|\boldsymbol{a}| \leqslant 1$ and $|\boldsymbol{b}| \leqslant 1$, then $|\boldsymbol{a}+\boldsymbol{b}|^{2} \leqslant(2-|\boldsymbol{a}-\boldsymbol{b}|)^{2}$, i.e.,

$$
\begin{aligned}
& |\boldsymbol{a}|^{2}+|\boldsymbol{b}|^{2}+2\langle\boldsymbol{a}, \boldsymbol{b}\rangle \\
& \leqslant 4-4|\boldsymbol{a}-\boldsymbol{b}|+|\boldsymbol{a}|^{2}+|\boldsymbol{b}|^{2}-2\langle\boldsymbol{a}, \boldsymbol{b}\rangle,
\end{aligned}
$$

which is equivalent to $|\boldsymbol{a}-\boldsymbol{b}| \leqslant 1-\langle\boldsymbol{a}, \boldsymbol{b}\rangle$. Then $(|\boldsymbol{a}-\boldsymbol{b}|)^{2} \leqslant(1-\langle\boldsymbol{a}, \boldsymbol{b}\rangle)^{2}$ implies that $|\boldsymbol{a}|^{2}+|\boldsymbol{b}|^{2} \leqslant$ $1+\langle\boldsymbol{a}, \boldsymbol{b}\rangle^{2}$.

From the above proposition, we see that $f(\boldsymbol{a}, \boldsymbol{b})>1$ if and only if $g(\boldsymbol{a}, \boldsymbol{b})>2$, namely, $\{(\boldsymbol{a}, \boldsymbol{b}): f(\boldsymbol{a}, \boldsymbol{b})>1\}=$ $\{(\boldsymbol{a}, \boldsymbol{b}): g(\boldsymbol{a}, \boldsymbol{b})>2\}$.

\section{Appendix B: PROBABILITY DENSITY FUNCTION OF THE INNER PRODUCT OF TWO INDEPENDENT RANDOM UNIT VECTORS}

Recall that there is a unique unitary-invariant measure (up to normalization) $\mu$ over the sphere:

$$
\mathrm{d} \mu(\boldsymbol{u})=\frac{1}{N_{m}} \delta(1-|\boldsymbol{u}|)[\mathrm{d} \boldsymbol{u}],
$$

where

$$
N_{m}=\int_{\mathbb{R}^{m}} \delta(1-|\boldsymbol{u}|)[\mathrm{d} \boldsymbol{u}]=\frac{2 \pi^{\frac{m}{2}}}{\Gamma\left(\frac{m}{2}\right)} .
$$

Now the probability density function of the inner product $\langle\boldsymbol{u}, \boldsymbol{v}\rangle$ between two independent random unit vectors $\boldsymbol{u}$ and $\boldsymbol{v}$ can be expressed as

$$
p_{m}(s)=\int \delta(s-\langle\boldsymbol{u}, \boldsymbol{v}\rangle) \mathrm{d} \mu(\boldsymbol{u}) \mathrm{d} \mu(\boldsymbol{v}) .
$$

By using the Haar measure (also denoted $\mu$ ) over the orthogonal group, the above integral can be rewritten as

$$
\begin{aligned}
p_{m}(s) & =\int \delta\left(s-\left\langle U \boldsymbol{e}_{1}, V \boldsymbol{e}_{1}\right\rangle\right) \mathrm{d} \mu(U) \mathrm{d} \mu(V) \\
& =\int \delta\left(s-\left\langle\boldsymbol{e}_{1}, W \boldsymbol{e}_{1}\right\rangle\right) \mathrm{d} \mu(W) \\
& =\int \delta\left(s-\left\langle\boldsymbol{e}_{1}, \boldsymbol{u}\right\rangle\right) \mathrm{d} \mu(\boldsymbol{u}),
\end{aligned}
$$

where $U, V$, and $W$ are some unitary operators. 
Using the Lebesgue measure, we obtain that

$$
\begin{aligned}
p_{m}(s) & =\frac{1}{N_{m}} \int \delta\left(s-u_{1}\right) \delta(1-|\boldsymbol{u}|)[\mathrm{d} \boldsymbol{u}] \\
& =\frac{2}{N_{m}} \int_{\mathbb{R}^{m-1}} \delta\left(\left(1-s^{2}\right)-\sum_{j=2}^{m} u_{j}^{2}\right) \prod_{j=2}^{m} \mathrm{~d} u_{j} .
\end{aligned}
$$

Set

$$
\psi(t)=\int_{\mathbb{R}^{m-1}} \delta\left(t-\sum_{j=2}^{m} u_{j}^{2}\right) \prod_{j=2}^{m} \mathrm{~d} u_{j} .
$$

Then its Laplace transform is given by

$$
L(\psi)(\omega):=\prod_{j=2}^{m} \int_{\mathbb{R}} e^{-\omega u_{j}^{2}} \mathrm{~d} u_{j}=\left(\frac{\pi}{\omega}\right)^{\frac{m-1}{2}} .
$$

Hence

$$
\psi(t)=L^{-1}\left(\left(\frac{\pi}{\omega}\right)^{\frac{m-1}{2}}\right)(t)=\frac{\pi^{\frac{m-1}{2}}}{\Gamma\left(\frac{m-1}{2}\right)} t^{\frac{m-3}{2}} .
$$

Therefore we have

Proposition B.1. The probability density function of the inner product between $\boldsymbol{u}$ and $\boldsymbol{v}$ is given by $p_{m}(s)=\frac{2}{N_{m}} \psi\left(1-s^{2}\right)$, that is,

$$
p_{m}(s)=C_{m} \cdot\left(1-s^{2}\right)^{\frac{m-3}{2}}, \quad s \in[-1,1],
$$

where $C_{m}=\frac{\Gamma\left(\frac{m}{2}\right)}{\sqrt{\pi} \Gamma\left(\frac{m-1}{2}\right)}$.

In particular, when $m=3, p_{3}(s)=\frac{1}{2}$ for $s \in[-1,1]$.

Finally, the integral mentioned in the text is formulated as

$$
\begin{aligned}
& \iint \Phi(\langle\boldsymbol{u}, \boldsymbol{v}\rangle) \delta(1-|\boldsymbol{u}|) \delta(1-|\boldsymbol{v}|)[\mathrm{d} \boldsymbol{u}][\mathrm{d} \boldsymbol{v}] \\
& =N_{m}^{2} \int_{-1}^{1} \Phi(s) p_{m}(s) \mathrm{d} s
\end{aligned}
$$

for any suitable function $\Phi(\cdot)$ of the inner product $\langle\boldsymbol{u}, \boldsymbol{v}\rangle$.

\section{Appendix C: CALCULATION OF THE VOLUME: $\operatorname{vol}\left(\Theta_{2,2}^{\mathrm{NJM}}\right)$}

In fact,

$$
\operatorname{vol}\left(\Theta_{2,2}^{\mathrm{NJM}}\right)=\int_{\Theta_{2,2}^{\mathrm{NJM}}} \mathrm{d} a_{0} \mathrm{~d} b_{0}[\mathrm{~d} \boldsymbol{a}][\mathrm{d} \boldsymbol{b}]
$$

where a generic element $\left(a_{0}, a \cdot \boldsymbol{u}, b_{0}, b \cdot \boldsymbol{v}\right)$ of the set $\Theta_{2,2}^{\mathrm{NJM}} \subset$ $\Theta_{2,2}$ should satisfy inequality (4). Thus $\Theta_{2,2}^{\mathrm{NJM}}$ can be transformed into the following form:

$$
\widetilde{\Theta}_{2,2}^{\mathrm{NJM}}=\left\{\left(a_{0}, a, b_{0}, b, s\right):\left\{\begin{array}{l}
\left|a_{0}\right|+a \leqslant 1,\left|b_{0}\right|+b \leqslant 1, \text { where } a_{0}, b_{0} \in[-1,1], a, b \in[0,1] \\
\left(1-h\left(a_{0}, a\right)^{2}-h\left(b_{0}, b\right)^{2}\right)\left(1-\frac{a_{0}^{2}}{h\left(a_{0}, a\right)^{2}}-\frac{b_{0}^{2}}{h\left(b_{0}, b\right)^{2}}\right)>\left(a b s-a_{0} b_{0}\right)^{2} \\
h\left(x_{0}, x\right)=\frac{\sqrt{\left(1+x_{0}\right)^{2}-x^{2}}+\sqrt{\left(1-x_{0}\right)^{2}-x^{2}}}{2} \text { and } s \in[-1,1]
\end{array}\right\} .\right.
$$

Based on this observation, we get

By numerical calculation we have $\operatorname{vol}\left(\Theta_{2,2}^{\mathrm{NJM}}\right) \doteq 1.09662$.

$$
\begin{aligned}
\operatorname{vol}\left(\Theta_{2,2}^{\mathrm{NJM}}\right) & =N_{3}^{2} \int_{\widetilde{\Theta}_{2,2}^{\mathrm{NJM}}} a^{2} b^{2} p_{3}(s) \mathrm{d} a_{0} \mathrm{~d} b_{0} \mathrm{~d} a \mathrm{~d} b \mathrm{~d} s \\
& =8 \pi^{2} \int_{\widetilde{\Theta}_{2,2}^{\mathrm{NJM}}} a^{2} b^{2} \mathrm{~d} a_{0} \mathrm{~d} b_{0} \mathrm{~d} a \mathrm{~d} b \mathrm{~d} s .
\end{aligned}
$$

[1] J.S. Bell, Physics 1, 195 (1964).

[2] H.M. Wiseman, S.J. Jones, and A.C. Doherty, Phys. Rev. Lett. 98, 140402 (2007).

[3] A. Einstein, B. Podolsky, N. Rosen, Phys. Rev. 47, 777 (1935).

[4] R.F. Werner, Phys. Rev. A40, 4277 (1989).

[5] J.S. Bell, Rev. Mod. Phys. 38(3), 447-452(1966).

[6] W. Heisenberg, Zeitschrift für Physik (in German) 43(3-4): 172-198 (1927).

[7] T. Baumgratz, M. Cramer, and M.B. Plenio, Phys. Rev. Lett. 113, 140401 (2014).
[8] P. Busch, P. Lahti, and R.F. Werner, J. Math. Phys. 55, 042111 (2014).

[9] R. Uola, C. Budroni, O. Gühne, and J.-P. Pellonpää, Phys. Rev. Lett. 115, 230402 (2015).

[10] A. Fine, Phys. Rev. Lett. 48, 291 (1982).

[11] M.M. Wolf, D. Perez-Garcia, and C. Fernandez, Phys. Rev. Lett. 103, 230402 (2009).

[12] E. Bene and T. Vértesi, New. J. Phys. 20, 013021(2018).

[13] M.T. Quintino, J. Bowles, F. Hirsch, and N. Brunner, Phys. Rev. A93, 052115 (2016). 
[14] F. Hirsch, M.T. Quintino, and N. Brunner, Phys. Rev. A97, 012129 (2018).

[15] A. Bluhm and I. Nechita, J. Math. Phys. 59, 112202 (2018).

[16] A. Bluhm and I. Nechita, arXiv: 1809.04514

[17] J. Jae, K. Baek, J. Ryu, and J. Lee, Phys. Rev. A 100, 032113 (2019).

[18] C. Carmeli, T. Heinosaari, and A. Toigo, Phys. Rev. Lett. 122, 130402 (2019).

[19] C. Carmeli, T. Heinosaari, and A. Toigo, Phys. Rev. A 98, 012126 (2018).

[20] P. Skrzypczyk, I. Šupić, and D. Cavalcanti, Phys. Rev. Lett. 122, 130403 (2019).

[21] R. Uola, T. Kraft, J. Shang, X-D. Yu, and O. Gühne, Phys. Rev. Lett. 122, 130404 (2019).

[22] K. Życzkowski, P .Horodecki, A. Sanpera and M. Lewenstein, Phys. Rev. A 58, 883 (1998).

[23] K. Życzkowski and H.J. Sommers, J. Phys. A : Math. Ge. 36,
10115 (2003).

[24] A. Andai, J. Phys. A : Math. Theor. 39, 13641 (2006).

[25] J.E. Avron, O. Kenneth, Ann. Phys. 324(2), 470-496 (2009).

[26] J. Samuel, K. Shivam, S. Sinha, Ann. Phys. 396, 159-172 (2018).

[27] P. Slater, J. Phys. A : Math. Theor. 46, 445302 (2013).

[28] A. Lovas and A. Andai, J. Phys. A : Mahth. Theor. 58, 295303 (2017).

[29] P. Busch, Phys. Rev. D 33, 2253 (1986).

[30] S. Yu, N.L. Liu, L. Li and C.H. Oh, Phys. Rev. A 81, 062116 (2010).

[31] L. Zhang, J. Wang, and Z. Chen, Phys. Lett. A 382 (23), 15161523 (2018).

[32] G. Aubrun and S.J. Szarek, Phys. Rev. A73(2), 022109 (2006).

[33] G. Aubrun, S.J. Szarek and D. Ye, Commun. Pure Appl. Math. 67(1), 1-52 (2014). 